\title{
Submitted: Infraspinatus atrophy due to Bennett lesion causing Accepted: suprascapular nerve palsy
} 24.03.2021

Published: 07.06.2021

\author{
Keywords \\ infraspinatus, \\ muscle atrophy, \\ suprascapular \\ nerve entrapment \\ syndrome, \\ Bennett lesion, \\ denervation
}

\author{
Juan Miranda Bautista¹, Javier Fernández-Jara², Santiago Miranda Bautista \\ Pablo Menéndez Fernández-Miranda ${ }^{4}$, María Valencia Mora ${ }^{5}$, \\ Begoña Gutiérrez San José6, Mateo González Estévez', \\ Blanca Mur Molina ${ }^{3}$, Patricia Patilla Vázquez ${ }^{1}$
}

${ }^{1}$ Department of Radiology, Hospital General Universitario Gregorio Marañón, Spain

${ }^{2}$ Department of Radiology, Hospital Universitario Rey Juan Carlos, Madrid, Spain

${ }^{3}$ Department of Rehabilitation, Hospital Universitario Ramón y Cajal, Madrid, Spain

${ }^{4}$ Department of Radiology, Hospital Universitario Marqués de Valdecilla, Santander, Spain

${ }^{5}$ Department of Traumatology, Hospital Universitario Fundación Jiménez Díaz, Spain

${ }^{6}$ Department of Radiology, Hospital Universitario Fundación Jiménez Díaz, Spain

Correspondence: Dr.Juan Miranda Bautista, e-mail:mirandabautistajuan@gmail.com

DOI: $10.15557 / \mathrm{JoU} .2021 .0028$

\begin{abstract}
We report the case of a 19-year-old professional volleyball player who presented with right shoulder pain exacerbated during sports activity. On physical examination, infraspinatus atrophy was evident. As the clinical setting suggested suprascapular nerve entrapment syndrome, shoulder MR and later CT were performed. The results showed radiological signs of subacute-chronic infraspinatus muscle denervation and a Bennett lesion of the shoulder, presumably due to chronic repetitive trauma during the classical overhead swing in volleyball. The patient agreed to surgical treatment, and arthroscopic decompression was achieved. After months of rehabilitation, the pain gradually subsided, the infraspinatus muscle recovered its trophism, and the patient progressively returned to her regular sports activity.
\end{abstract}

\section{Case report}

A 19-year-old professional volleyball player presented to the emergency department complaining of long-standing and progressively worsening right shoulder pain, which exacerbated during sports activity.

Physical examination revealed right subtle supraspinatus and obvious infraspinatus muscle atrophy. The range of motion was preserved, and there were no signs of instability or muscle weakness. There was no neck pain, numbness, or tingling in her arm or any previous shoulder dislocations or subluxations.

As the clinical setting suggested suprascapular nerve entrapment syndrome, conventional shoulder MR and later CT examinations were performed to confirm the diagnosis and look for etiology.
MR results (Fig. 1, Fig. 2) revealed atrophy and fatty infiltration as well as mild muscle edema, depicted on watersensitive sequences, features consistent with subacutechronic denervation of the infraspinatus muscle. Isolated denervation of the infraspinatus muscle oriented that the nerve impingement was located at the spinoglenoid notch. A bony prominence along the posterior glenoid rim was also detected.

CT images showed the osseous shoulder anatomy and glenoid deformity (Fig. 3), and a Bennett lesion was clearly identified as a curvilinear ossification along the posterior glenoid rim (at 9 o'clock).

The patient was finally diagnosed as having suprascapular nerve palsy secondary to a Bennett lesion causing isolated infraspinatus muscle atrophy. She agreed to surgical treatment, and arthroscopic decompression was accomplished. 


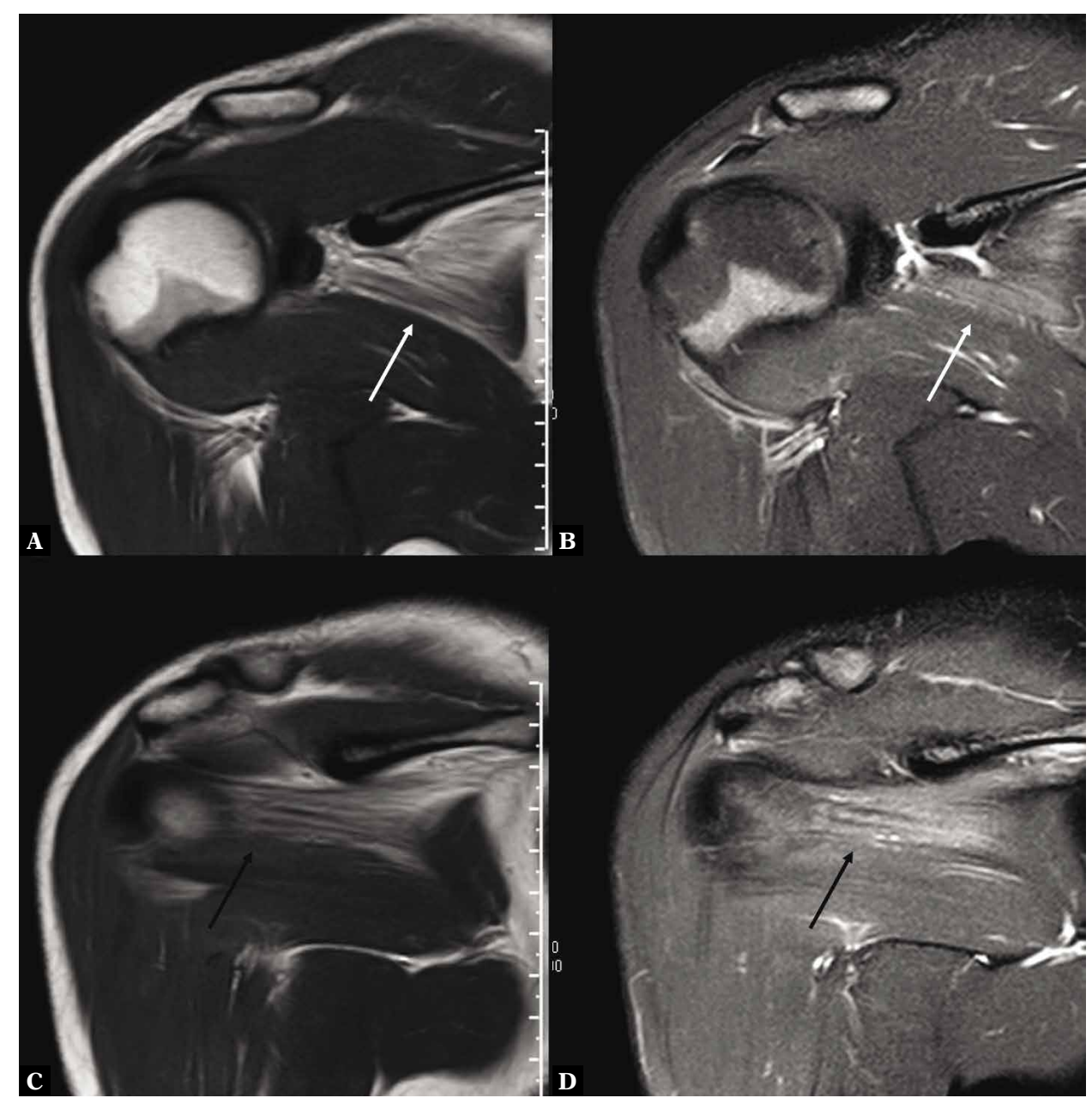

Fig. 1. Coronal T1 (A, C) and T2 (B, D) MR weighted images demonstrate muscle atrophy, discrete edema and fatty infiltration as radiological signs of subacute-chronic infraspinatus muscle denervation (white and black arrows)

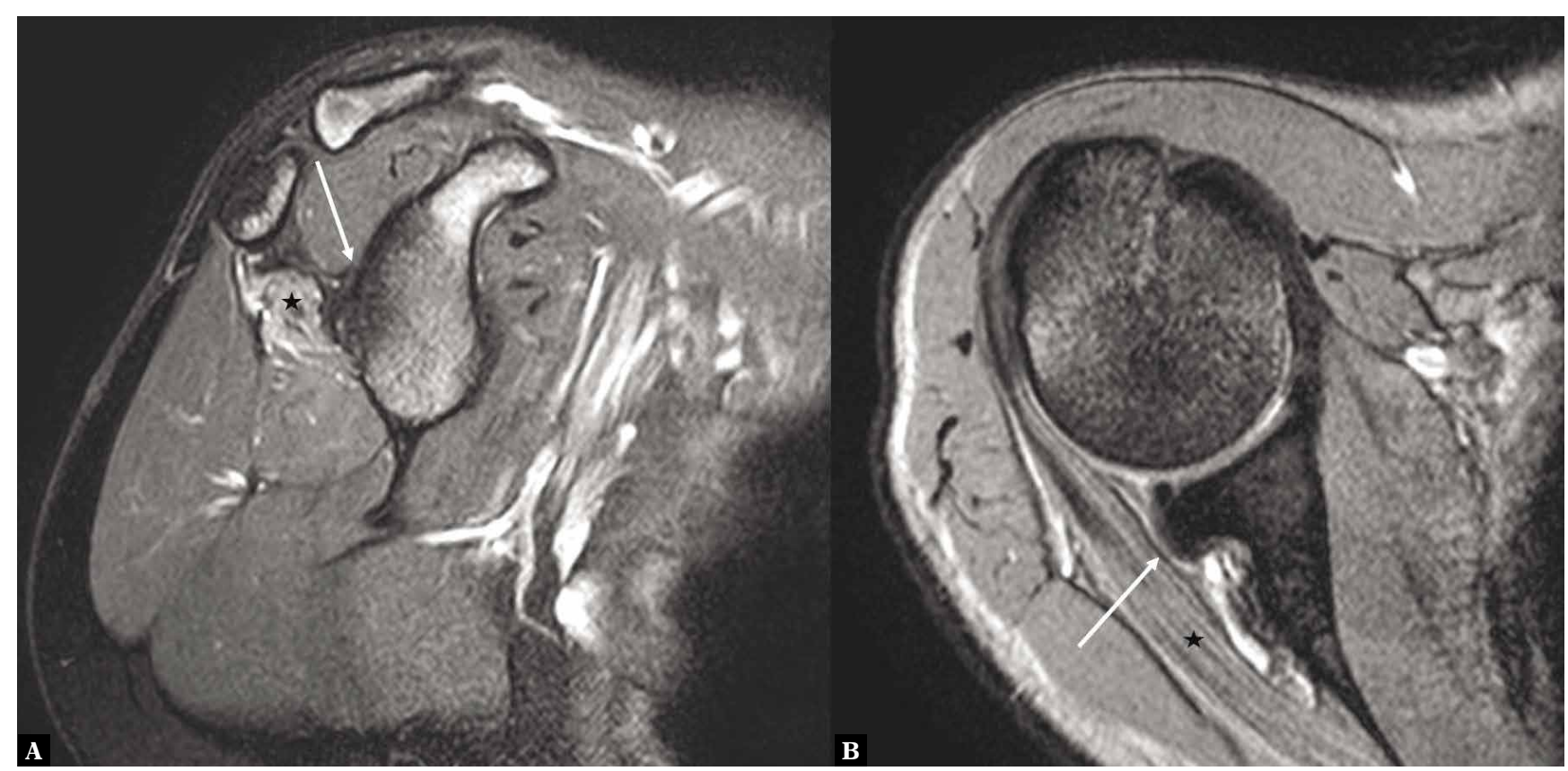

Fig. 1. Sagittal oblique (A) and axial (B) T2 MR weighted images show a bony prominence along the posterior glenoid rim and adjacent capsule-labral soft tissues (white arrows) and the infraspinatus muscle atrophy and fatty infiltration (black stars) 


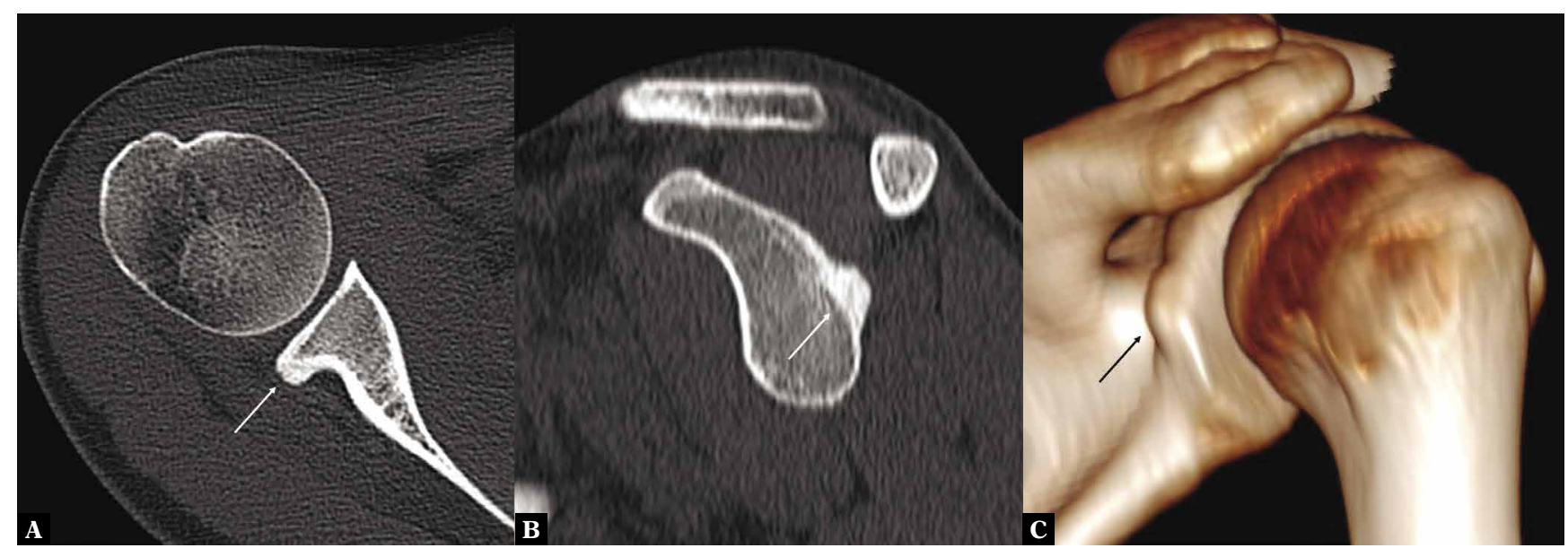

Fig. 3. Axial (A), sagittal (B) shoulder TC and 3D reconstructions (C) show an eccentric curvilinear ossification along the posterior glenoid rim (at $9 o^{\prime}$ clock) and adjacent capsule-labral soft tissues. The features are consistent with Bennett lesion of the shoulder

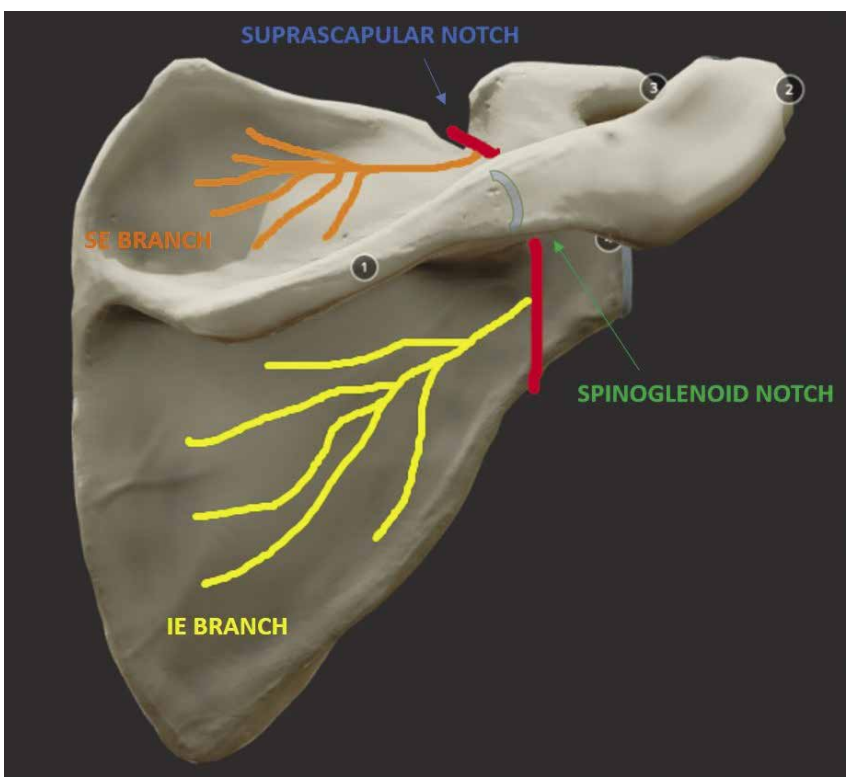

Fig. 4. Schematic representation of the suprascapular nerve on its course through the suprascapular notch (blue arrow) and the spinoglenoid notch (green arrow), giving its two main muscular branches for the supraspinatus (orange) and the infraspinatus (yellow) muscles. Spine of scapula (1), acromion (2), coracoid process (3), posterior glenoid rim (4)

After months of rehabilitation, the pain gradually subsided, the infraspinatus muscle recovered its trophism, and the patient progressively returned to her regular sports activity.

\section{Discussion}

Entrapment neuropathy secondary to nerve compression by mechanical or dynamic forces may cause upper extremity pain and weakness in athletes.

The suprascapular nerve arises from the upper trunk of the brachial plexus from the fifth and sixth nerve roots. It runs loosely under the trapezius muscle, forming a concave arch behind the clavicle. It enters the suprascapular notch
(Fig. 4, Fig. 5) beneath the superior transverse scapular ligament through the suprascapular foramen. The suprascapular nerve then enters the supraspinatus fossa and branches to the supraspinatus muscle. Finally, it curves around the spinoglenoid notch (Fig. 4, Fig. 6) and supplies the infraspinatus muscle $\mathrm{e}^{(1-3)}$.

The suprascapular nerve branches at the glenoid notch supplying independent innervation to the supraspinatus and infraspinatus muscles. The clinical and imaging manifestations of entrapment neuropathies of the suprascapular nerve and its branches vary depending on the location of compression. Entrapment of the suprascapular nerve at the scapular incisura results in supraspinatus and infraspinatus muscle denervation. In contrast, injury to the nerve at or below the spinoglenoid notch results in infraspinatus muscle atrophy with sparing of the supraspinatus muscle $^{(2,3)}$.

Ultrasound plays an important role in the initial assessment of shoulder pain and weakness. Although it was not initially performed in our patient, there are two main reasons why it can help in the diagnostic work-up of suprascapular nerve entrapment.

Firstly, the suprascapular and spinoglenoid notches can be easily assessed using this imaging technique. The suprascapular incisura, deep into the supraspinatus fossa, helps as a reference to locate the former (Fig. 5). The posterior aspect of the glenoid and the base of the scapular spine help to identify the latter (Fig. 6). However, due to the small size and deep location of this nerve in both notches, it is not identified unless there is a pathological enlargement. The vessel signal on color Doppler ultrasound can be advantageous in the identification of the neurovascular bundle location.

Secondly, ultrasound has also developed into an accepted and useful tool for evaluating rotator cuff patterns of muscle atrophy and fatty infiltration. In the absence of rotator cuff tendinopathy, these signs should raise a suspicion of peripheral neuropathy. 


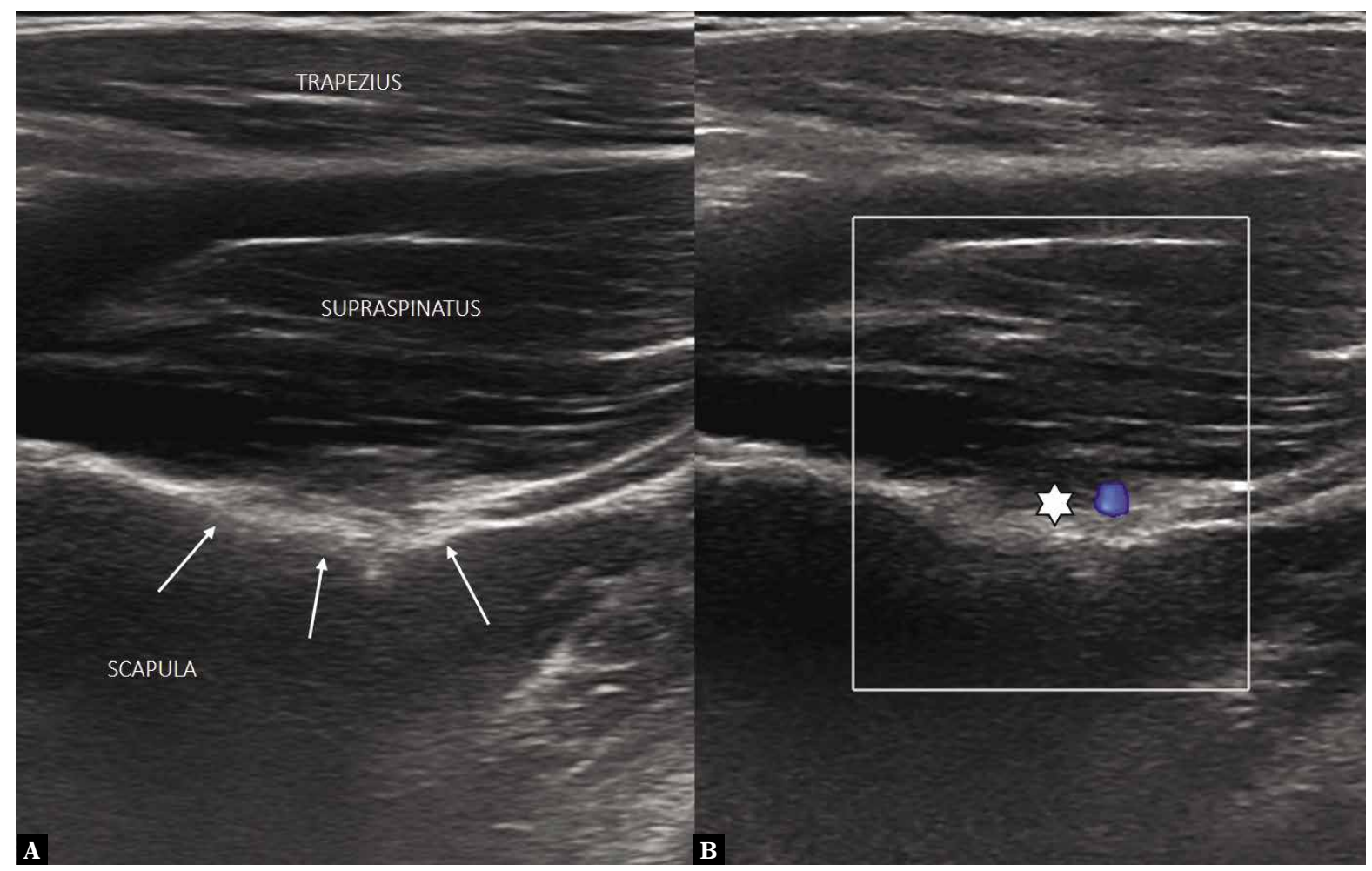

Fig. 5. Greyscale (A) ultrasound image depicts the anatomy near the suprascapular notch (white arrows in $\mathbf{A}$ ). Color Doppler (B) ultrasound image highlights the suprascapular neurovascular bundle (white star) deep in location to the trapezius and supraspinatus muscles. Suprascapular vessels serve as a reference in Color Doppler to locate the respective suprascapular nerve, which, unless pathologically enlarged, is not observed in conventional images

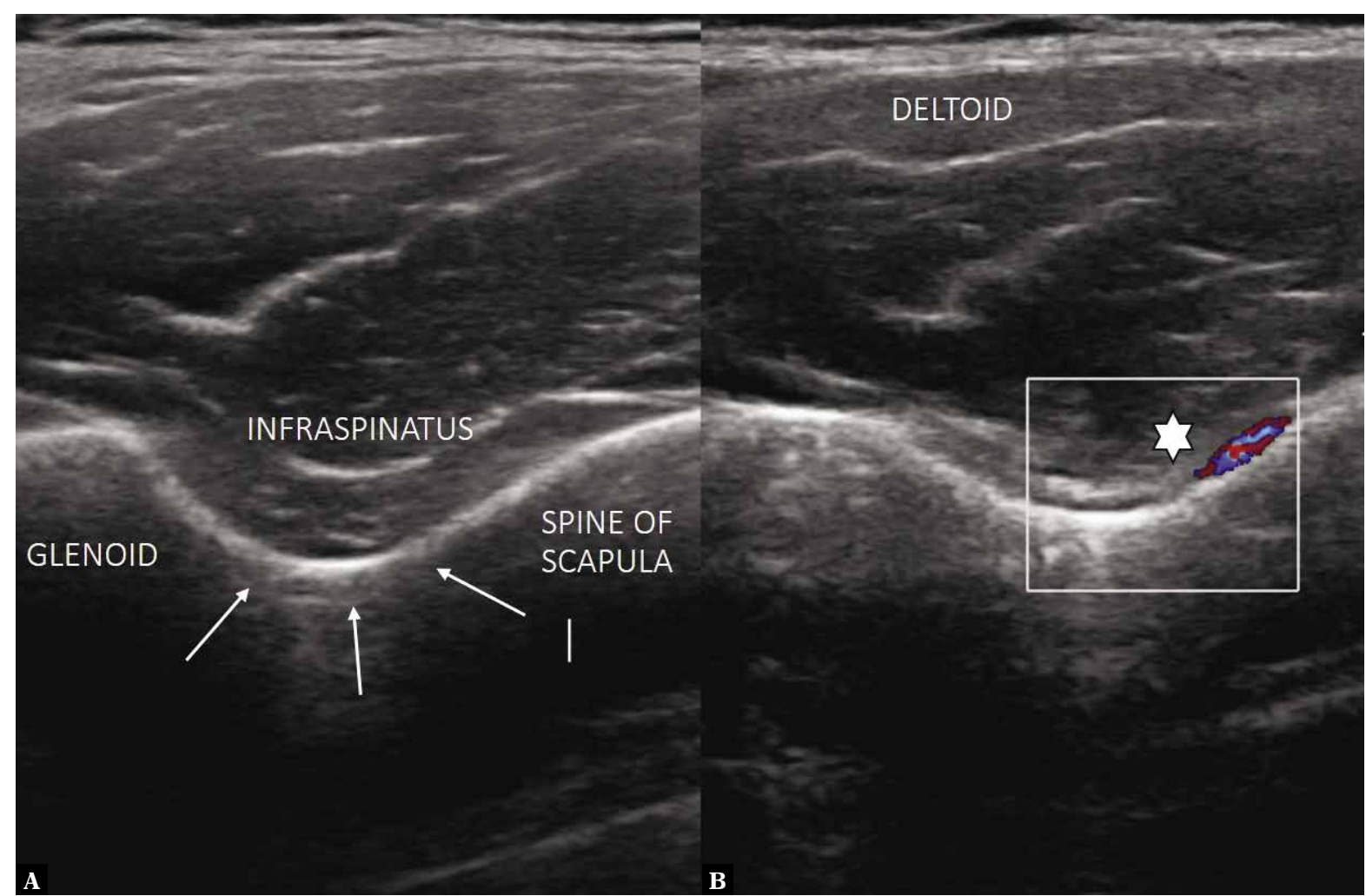

Fig. 6. Greyscale (A) ultrasound image shows the anatomy near the spinoglenoid notch (white arrows in A). Color Doppler (B) ultrasound image highlights the suprascapular neurovascular bundle (white star) in the deep surface of the spinoglenoid notch. Suprascapular vessels serve as a reference to locate the respective suprascapular nerve 
Suprascapular nerve entrapment syndrome has a large number of well-known causes. It may be due to compression from space-occupying lesions, including labral or ganglion cysts, hematomas, enlarged varicosities, malignant tumors, or it may be iatrogenic ${ }^{(1,4,5)}$. Greyscale and Doppler ultrasound may be the first imaging techniques to identify and diagnose the source of symptomatology. Moreover, it can also be used as a guide for diagnostic and therapeutic procedures, such as ultrasound-guided biopsy of solid masses, aspiration of ganglion cysts, draining of hematomas, intraarticular injection of corticosteroids, or suprascapular nerve block ${ }^{(6-8)}$. In almost any patient, complementary CT or MR are recommended in order to rule out bony causes - as in the present case - and better visualize the underlying extension of the pathology.

Chronic traumatic causes of suprascapular nerve entrapment are also common. Sometimes they are due to repetitive strain on the abducted and externally rotated shoulder, leading to a thickened transverse scapular ligament ${ }^{(9)}$. Additionally, repetitive overhead activities and forceful rotational movements performed during sports such as baseball, volleyball, and weightlifting, may be the cause of a Bennett lesion, such as that described in our case. It refers to an ossification of the posteroinferior glenoid in the proximity of the posterior band of the inferior glenohumeral ligament complex. In volleyball players, Bennett lesions tend to occur more frequently during the overhead swing, when the humeral head is forced posteriorly while in maximum external rotation during the cocking and extension phases ${ }^{(10)}$. Importantly, a study conducted in Austria demonstrated atrophy of the infraspinatus muscle in $30 \%$ of the hitting shoulders of beach volley players, and the condition was not typically recognized by the players ${ }^{(11)}$.

\section{References}

1. Linda DD, Harish S, Stewart BG, Finlay K, Parasu N, Rebello RP: Multimodality imaging of peripheral neuropathies of the upper limb and brachial plexus. Radiographics 2010; 30: 1373-400.

2. Bencardino JT, Rosenberg ZS: Entrapment neuropathies of the shoulder and elbow in the athlete. Clin Sports Med 2006; 25: 465-487, vi-vii.

3. Ferretti A, De Carli A, Fontana M: Injury of the suprascapular nerve at the spinoglenoid notch. Am J Sports Med 1998; 26: 759-763.

4. Fehrman DA, Orwin JF, Jennings RM: Suprascapular nerve entrapment by ganglion cysts: a report of six cases with arthroscopic findings and review of the literature. Arthroscopy 1995; 11: 727-734.

5. Yanny S, Toms AP: MR patterns of denervation around the shoulder. AJR Am J Roentgenol 2010; 195: W157-163.

6. Hashimoto BE, Hayes AS, Ager JD: Sonographic diagnosis and treatment of ganglion cysts causing suprascapular nerve entrapment. J Ultrasound Med 1994; 13: 671-674.

7. Tagliafico A, Russo G, Boccalini S, Michaud J, Klauser A et al.: Ultrasound-guided interventional procedures around the shoulder. Radiol Med 2014; 119: 318-326.
The treatment of infraspinatus atrophy due to suprascapular nerve compression depends on the duration of symptoms, location and cause of the entrapment. Spontaneous recovery can occur with the strengthening of the external rotators and cessation of exacerbating activities. Surgical decompression is indicated depending on demands and patients' symptomatology. Other etiologies for compression neuropathies that would also warrant surgical removal include labral cysts, ganglion from a labral tear, and other mass lesions ${ }^{(3,5,12,13)}$.

\section{Conclusions}

Bennett lesion of the shoulder is an uncommon but characteristic pathologic entity in throwing sports like volleyball, and a potential cause of isolated infraspinatus muscle denervation due to entrapment of the suprascapular nerve at the spinoglenoid notch. Ultrasound can serve as the firstline imaging modality to rule out other causes of nerve entrapment at the suprascapular and spinoglenoid notches, such as space-occupying lesions. Moreover, it can help as a guide for many therapeutic procedures. However, complementary CT or MR are recommended to look for bony lesions and better visualize the underlying extension of the pathology.. Both the clinical context and imaging modalities are helpful in achieving the final diagnosis and, consequently, contribute to better patient care and prognosis.

\section{Conflict of interest}

The authors do not report any financial or personal connections with other persons or organizations, which might negatively affect the contents of this publication and/or claim authorship rights to this publication.

8. Bianchi S, Becciolini M, Urigo C: Ultrasound imaging of disorders of small nerves of the extremities: less recognized locations. J Ultrasound Med 2019; 38: 2821-2842.

9. Carroll KW, Helms CA, Otte MT, Moellken SM, Fritz R: Enlarged spinoglenoid notch veins causing suprascapular nerve compression. Skeletal Radiol 2003; 32: 72-77.

10. Pohlgeers KM, Becker JA: Infraspinatus atrophy in a volleyball player: a case of a bennett lesion causing nerve impingement. Curr Sports Med Rep 2014; 13: 358-360.

11. Lajtai G, Pfirrmann CW, Aitzetmüller G, Pirkl C, Gerber C et al.: The shoulders of professional beach volleyball players: high prevalence of infraspinatus muscle atrophy. Am J Sports Med 2009;37: 1375-1383.

12. McCluskey L, Feinberg D, Dolinskas C: Suprascapular neuropathy related to a glenohumeral joint cyst. Muscle Nerve 1999; 22: 772-777.

13. Osterwalder C, Meyer VE: Trans-trapezial approach to the incisura scapulae in incisura scapulae syndrome: an anatomic study [in German]. Handchir Mikrochir Plast Chir 1990; 22: 115-119. 